BULL. AUSTRAL. MATH. SOC.

VOL. $29(1984), 101-108$.

\title{
SOME SUGGESTIONS FOR FURTHER RESEARCH
}

\author{
KURT MAHLER
}

At the age of $80 \mathrm{I}$ cannot expect to do much more mathematics. I may, however, state a number of questions where perhaps further research might lead to interesting results.

\section{A problem on Liouville numbers}

Long ago, Maillet [9] proved that if $X$ is a Liouville number, and $f(z)$ is a rational function with rational coefficients, then also $f(X)$ is a Liouville number. This leads me to ask the following question.

Which analytic functions $f(z)$ have the property that if $X$ is any Liouville number, then so is $f(X)$ ? In particular, are there entire transcendental functions with this property? The difficulty of this problem lies of course in the fact that the set of all Liouville numbers is non-enumerable.

\section{Cantor's set}

Cantor's set $C$ consists of all numbers

$$
X=\sum_{h=1}^{\infty} 2 d_{h} 3^{-h}
$$

where all the coefficients $d_{h}$ are either 0 or 1 , thus the products $2 d_{h}$ are either 0 or 2 . The related numbers

Received 27 July 1983.

Copyright Clearance Centre, Inc. Serial-fee code: 0004-9727/84 $\$ A 2.00+0.00$. 


$$
x=\sum_{h=1}^{\infty} d_{h} 2^{-h}
$$

form the closed interval $c=[0,1]$. We write $X=M(x)$. Whenever $X$ is rational, so is $x$, and vice versa.

If $X$ and $x$ are rational, the sequence $d_{1}, d_{2}, d_{3}, \ldots$ is periodic, say of period length $P$ and with $p$ terms before the start of the periodic part. Then two numerators $N$ and $n$ exist such that

$$
X=N / 3^{p}\left(3^{P}-1\right) \text { and } x=n / 2^{P}\left(2^{P}-1\right) \text {, }
$$

where the numerators and the denominators need not be relatively prime. Here the difficult problem of the simultaneous factorisation of

$$
3^{P}-1 \text { and } 2^{P}-1
$$

for the same $P$ plays a role if both $X$ and $x$ are to be written as reduced fractions. While in many examples the exact denominator of $X$ is larger than that of $x$, there are also cases when the opposite is true.

Next let both $X$ and $x$ be irrational, $X=M(x)$. The best rational approximations of $\boldsymbol{x}$ are the convergents,

$$
u_{p} / v_{r}=n_{r} / 2^{P_{r}}\left(2^{P_{r}}-1\right) \quad \text { say }
$$

of the continued fraction of $x$. The images under $M$,

$$
U_{p} / V_{r}=M\left(u_{r} / v_{r}\right)=N_{r} / 3^{p} r\left(3^{P} r_{-1}\right),
$$

are still good approximations of $X$, but need not be convergents of the continued fraction for $X$. Nor need the convergents of the continued fraction for $X$ lie in Cantor's set. Thus the following problem arises.

How close can irrational elements of Cantor's set be approximated by rational numbers

(i) in Contor's set, and

(ii) by rational numbers not in Cantor's set?

A second problem is much more difficult. It asks the following question.

Are irrational elements of Cantor's set necessarily 
transcéndental? Thus does Cantor's set contain no irrational algebraic elements?

A possible approach to this question consists in the study of the nonhomogeneous linear expressions

$$
\left|3^{p} r^{+P} r_{X}-3^{p} r_{X}-N_{r}\right|
$$

It may be that a p-adic form of Schmidt's theorem on the rational approximations of algebraic numbers [10] holds for such expressions.

\section{A non-linear functional equation}

Let $q \geq 2$ be an integer and $P(u, v)$ an irreducible polynomial with complex coefficients which contains both variables $u$ and $v$ to positive degrees. Recently, in a paper which is yet to appear, I gave necessary and sufficient conditions under which the functional equation

$$
P\left(f(z), f\left(z^{q}\right)\right)=0
$$

has a non-constant analytic solution $f(z)$ which is either regular in a neighbourhood of the point $z=0$ or has a pole at this point. The functional equation ( $P$ ) enables us to continue this function into the unit disk $|z|<1$, but $f(z)$ need no longer be single-valued in this disk, but may have infinitely many algebraic branch points.

The following question is therefore appropriate.

Under which conditions on $q$ and $P(u, v)$ has the fronctional equation (P) a solution $f(z)$ which is regular and single valued in the region $0<|z|<1$, but which has an essential singularity at $z=0$ ?

I conjecture that a suitable change of variable will lead to a solution.

\section{Fourier coefficients of modular forms}

In my paper (Mahler [8]) I applied the transformation theory of modular functions to establish simple systems of recursive formulae for the Fourier coefficients of such functions, for example, for $j(\omega)$ and $j(\omega)^{1 / 3}$. The method was purely algebraic. 
Can this method be generalised so as to lead to analogous recursive formulae for the Fourier coefficients of modular forms?

\section{Functions algebraic at algebraic points}

Let $\Sigma$ and $\Sigma_{0}$ be the sets of all meromorphic transcendental, and of all rational, functions $f(z)$ with the following property.

If $\alpha$ is any algebraic number, then $f(z)$ has in a neighbourhood of $z=\alpha$ either a power series

$$
f(z)=\sum_{h=0}^{\infty} f_{h}(z-\alpha)^{h}
$$

or a Laurent series

$$
f(z)=\sum_{h=-n}^{\infty} f_{h}(z-\alpha)^{h}
$$

in which all the coefficients $f_{h}$ are algebraic numbers.

It was a classical result by Faber ([3]; see also Mahler [7]) that the set $\Sigma$ is not empty and even contains entire transcendental functions. It is further clear that if $R\left(z, w_{1}, \ldots, w_{p}\right)$ is a polynomial with algebraic coefficients and if $f_{1}(z), \ldots, f_{p}(z)$ are any functions in $\Sigma$, then

$$
P\left(z, f_{1}(z), \ldots, f_{p}(z)\right)
$$

is an element of either $\Sigma$ or of $\Sigma_{0}$; further, with every function in $\Sigma$ also all its derivatives belong to $\Sigma$.

I propose the following problem.

Can a function $f(z)$ in $\Sigma$ satisfy an algebraic differential equation?

\section{Applications of ceilings}

In my paper (Mahler [6]) I introduced the notion of ceilings in algebraic number fields and used it to establish general lower and upper bounds for all the valuations of all the basis elements of any fractional 
divisor or ideal of the field. I now suggest the following investigations.

Let $K$ and $L$ be two finite algebraic number fields over $Q$ where $K$ is a proper subfield of $L$. Study the relations between the ceilings $\lambda(\underline{p})$ of $K$ and the ceilings $\Lambda(\underline{\underline{P}})$ of $L$, and deduce inequalities linking the bases of ideals a in $K$ with the bases of ideals $\underline{\underline{A}}$ in $L$. Apply the results to the study of relative algebraic number fields.

Investigate the bases of the differents of $K$ and $L$.

Use my general results on compound convex bodies (Mahler [4]) and on invariont matrices (Mahler [5]) to study the ideal bases in fields $Q\left(\theta, \theta^{\prime}, \ldots, \theta^{(k-1)}\right)$ obtained by adjoining to the rational field more than one root of an irreducible polynomial in $Q[x]$.

\section{On a recursively constructed set of points}

In the paper (Billing and Mahler [1]) the following recursive construction of a set of points was given.

In the projective plane denote by $\left[k, k^{\prime}\right]$ the line through two distinct points $k$ and $k^{\prime}$, and by $\left(L, L^{\prime}\right)$ the point of intersection of two distinct lines $L$ and $L^{\prime}$.

Let now $0,1,2,3$, and 4 be five distinct points no three of which are collinear. Define five further points $-1, \ldots,-5$ by

$$
\begin{gathered}
-3=([0,3],[1,2]),-4=([0,4],[1,3]), \\
-5=([1,4],[2,3]),-1=([0,1],[-3,4]),-2=([0,2],[-1,3]),
\end{gathered}
$$

then for every integer $k \geq 5$ the point $k$ as the intersection of all the lines

$$
\left[k^{\prime}-k,-k^{\prime}\right] \text { where } 0 \leq k^{\prime}<k / 2 \text {, }
$$

and for every integer $k \geq 6$ the point $-k$ as the intersection of all the lines

$$
\left[k-k^{\prime}, k^{\prime}\right] \text {, where } 1 \leq k^{\prime}<k / 2 \text {. }
$$

Then a sufficient condition for three points $k, k^{\prime}, k^{\prime \prime}$ to be collinear is that $k+k^{\prime}+k^{\prime \prime}=0$, but this condition need not be necessary if the original points $0,1, \ldots, 4$ are in special positions.

This construction leads to a sequence of points $k$ on a cubic, which 
may possibly be degenerate. If it is of genus 1 , then the coordinates of the point $k$ take the form of elliptic functions $\phi_{j}(k \alpha), j=1,2,3$, all with the same periods; $\alpha$ is a certain number which is not a period. If no rational multiple of $\alpha$ is a period, then the points $k$ are all distinct, and in the case of a real cubic lie dense on its infinite branch.

Can an analogous construction (which, however, may be algebraic of higher order) be given for sets of $p$ points on algebraic curves of genus $p>1$ ? Can something similar be done, say for Kummer's surface which can be defined by hyperelziptic fronctions of genus 2 ?

\section{Second order Farey polynomials}

In the paper (Brown and Mahler [2]) the following generalisation of the well known Farey sequences was introduced.

Let $n$ be a positive integer, $G_{n}$ the set of all polynomials

$$
a x^{2}+b x+c=[a, b, c]
$$

with integral coefficients $a, b, c$ satisfying

$0 \leq a \leq n,-n \leq b \leq n,-n \leq c \leq n \quad$ (when $a=0$, then $b \geq 1$ )

and let $F_{n}$ be the subset of $G_{n}$ which consists of all polynomials in $G_{n}$ which have either two real irrational zeros, or are linear and so have just one rational zero. Form the set of all the zeros of all the polynomials in $F_{n}$ and number these in order of increasing size; apply the same ordering also to the polynomials in $F_{n}$ so that the quadratic polynomials occur twice and the linear ones only once. In the lowest case $n=1$ we so so obtain the sequence

$$
\begin{array}{r}
{[1,1,-1],[0,1,1],[1,-1,-1],[0,1,0],} \\
{[1,1,-1],[0,1,-1],[1,-1,-1]}
\end{array}
$$

with the corresponding zeros

$$
-1.618 \ldots,-1,-0.618 \ldots, 0,0.618 \ldots, 1,1.618 \ldots .
$$

$F_{2}$ contains 31 polynomials, $F_{3}$ contains $103, F_{4}$ contains 223 ,

$F_{5}$ contains $483, F_{6}$ contains $763, F_{7}$ contains 1311 polynomials, 
and so on.

Associate with each triplet of three consecutive polynomials in $F_{n}$ their determinant. Then the original calculations suggested that, if the polynomial in the middle was not linear, then this determinant has one of the three values $-1,0$, or +1 ; other values could however be obtained when the polynomial in the middle was linear.

When Brown's calculations were extended here at Canberra, it turned out that already for $n=7$ the determinant could have values distinct from $-1,0$, and +1 even when the middle polynomial was quadratic; the following exceptions were obtained:

$$
\begin{aligned}
& {[1,6,7],[1,3,-6],[2,7,-7] \text {, determinant }-2,} \\
& {[7,-7,-2],[6,-3,-1],[7,6,1] \text {, determinant }-2,}
\end{aligned}
$$

and once more the same triplets of polynomials in reversed order for their second zeros.

The number of exceptional triplets increases with $n$, but I do not know how quickly.

Let us therefore propose the following investigations.

Denote by

$$
x \rightarrow \frac{a x+b}{c x+d} \quad(a d-b c= \pm 1)
$$

a fixed modular transformation, and let $n$ be very large. Are there sections of $F_{n}$ which are transformed into each other by such a transformation? (This is certainly so for $x \rightarrow-x$ and $x \rightarrow-x^{-1}$ ). How are the elements of $F_{n+1}$ not in $F_{n}$ distributed amongst the elements of $F_{n} ?$

Study the general low of the determinants of triplets of consecutive polynomials in $F_{n}$.

If $-1 \leq X \leq+1$, how close can $X$ be approximated by elements of $F_{n} ?$ 


\section{References}

[1] G. Billing and K. Mahler, "On exceptional points on cubic curves", $J$. London Math. Soc. 15 (1940), 32-43.

[2] A. Brown and K. Mahler, "A generalization of Farey sequences: some exploration via the computer", J. Number Theory 3 (1971), 364-370.

[3] Georg Faber, "Über arithmetische Eigenschaften analytischer Funktionen", Math. Ann. 58 (1904), 545-557.

[4] Kurt Mahler, "On compound convex bodies (I)", Proc. London Math. Soc. (3) 5 (1955), 358-379.

[5] K. Mahler, "Invariant matrices and the geometry of numbers", Proc. Roy. Soc. Edinburgh Sect. A 64 (1953-1957), 223-238.

[6] K. Mahler, "Inequalities for ideal bases in algebraic number fields", J. Austral. Math. Soc. 4 (1964), $425-448$.

[1] Kurt Mahler, Lectures on transcendental numbers (Lecture Notes in Mathematics, 546. Springer-Verlag, Berlin, Heidelberg, New York, 1976).

[8] K. Mahler, "On a class of non-linear functional equations connected with modular functions", J. Austral. Math. Soc. Ser. A 22 (1976), 65-118.

[9] E. Maillet, Introduction à Za théorie des nombres transcendants et des propriétés arithmétiques des fonctions (Gauthier-Villars, Paris, 1906).

[10] Wolfgang M. Schmidt, Diophantine approximation (Lecture Notes in Mathematics, 785. Springer-Verlag, Berlin, Heidelberg, New York, 1980).

Department of Mathematics, Institute of Advanced Studies, Australian National University, GPO Box 4, Canberra, ACT 260I, Australia. 